\title{
Use of High-fidelity Simulation Manikins to Teach Pharmacology to Pharmacy Undergraduates
}

\author{
Jamie J Coleman ${ }^{1,2,3}$, Sumaiyah Ali ${ }^{1}$, Christine Hirsch ${ }^{1}$, Julie Mason ${ }^{1}$ \\ ${ }^{1}$ Institute of Clinical Sciences, University of Birmingham, UK, ${ }^{2}$ University Hospitals Birmingham NHS Foundation \\ Trust, UK, ${ }^{3}$ West Midlands Centre for Adverse Drug Reactions, Sandwell and West Birmingham Hospitals NHS Trust, \\ $U K$
}

\section{Background}

Principles of pharmacology form the basis of many healthcare professional undergraduate curricula. They are essential for pharmacists who need this knowledge to inform their practice. Pharmacology has traditionally been taught using a didactic approach. High-fidelity simulation manikins are commonly used to train healthcare professionals but there are limited reports of use in pharmacy training or to reinforce core principles of pharmacology. This feasibility study examined the use of a high-fidelity simulation manikin (SimMan3G) to support teaching of pharmacology principles to undergraduate pharmacy students.

Methods

A total of 24 pharmacy students (years 2 and 3 of a four year programme) participated in a pharmacology simulation using a SimMan3G. The simulation centred on opioid analgesia and management of overdose with reference to the theories of receptor pharmacology. All students had received underpinning lecture-based introductions to these concepts as part of their degree. Student knowledge was determined with a 12 question pre- and post-session test based on defined learning objectives. A paired t-test was used for analysis of pre- and post-test scores. An evaluation of the session was conducted with all participants.

Results

Average scores for pre- and post-session tests were $52 \%$ and $90 \%$ respectively demonstrating a mean overall improvement of $38 \%(\mathrm{p}<0.01)$. In particular, knowledge of the monitoring parameters for and physiological signs of opioid overdose improved by $25 \%$ and $57 \%$ respectively. All participants agreed that simulation using a high-fidelity manikin was a useful addition to teaching and made subject matter more interesting. Regarding basic pharmacology, 100\% of participants felt that the simulation aided knowledge application and $96 \%$ felt that it would help them retain this knowledge.

Conclusions

This study demonstrates the feasibility of using high-fidelity simulation manikins and provides a template for successful integration into pharmacology teaching for undergraduate healthcare professionals. Manikin-based simulation in pharmacology was well received and improved student's short-term knowledge. We surmise that a mixture of practical and visual learning promoted a deeper understanding of therapeutic principles by offering an immersive experience that reinforced traditional lecture-based learning. Future work will look to confirm these pilot findings and assess long-term knowledge retention. 